\title{
Study of Spectral Components of Ventricular Repolarization Variability by Multiple Correspondence Analysis
}

\author{
S Wong ${ }^{1,2}$, AI Hernández ${ }^{2}$, F Carré ${ }^{2}$, G Carrault ${ }^{2}$ \\ ${ }^{1}$ Universidad Simon Bolivar, Caracas, Venezuela \\ ${ }^{2}$ Université de Rennes I, Rennes, France
}

\begin{abstract}
In this study we propose a novel approach, based on multiple correspondences analysis (MCA), to identify the relationships between the spectral variables obtained from HRV signals and those from ventricular repolarization variability (VRV). Parameters were extracted from $R R$ and $R T$ time series from 47 ECG records from aerobic and anaerobic sportsmen and sedentary subject. These numerical variables were transformed in categorical variables using a 7 classes fuzzy coding and several MCA were carried out. Results show that: i) aerobic disciplines present more variability than anaerobic ones, ii) mean RT interval are longer in sportsmen than in control subjects, iii) only the low frequencies of the HRV and VRV series are correlated, evidencing the heart rate modulation on ventricular repolarization, iv) the high frequencies of the $R T$ space seem to be inadequate markers for the characterization of the ANS modulation on ventricular repolarization.
\end{abstract}

\section{Introduction}

In order to understand the mechanisms of regulation of the ventricular activity by the autonomic nervous system (ANS), Heart Rate Variability (HRV) analysis techniques have been transposed to explore the ventricular repolarization variability (VRV). Spectral analysis of the VRV signal has shown the existence of two components, low frequency (LF) and high frequency (HF), which are consistent with LF and HF components observed in HRV $[1,2]$. Some works have sought to establish relations between the variability of the ventricular repolarization and the variability of the heart rate. Merri [1] conceived a night/day experiment to study the effects of the autonomic nervous system (ANS) on the duration of ventricular repolarization, on healthy subjects. It was found that spectral peaks on the LF and HF bands of the HRV and VRV series were very well correlated. Furthermore, the phase-delay between HRV and VRV were low in the LF band and almost zero in the HF band. These results suggest that there are oscillations in the VRV which are synchronous or quasi synchronous with those of the HRV and thus related to stimulations of the ANS. This means that a great part of the variability of the ventricular repolarization can be explained by the variability of the RR. Nollo [2] carried out a similar experience on 21 healthy subjects. Peaks in the LF and HF bands were also observed in the power spectra of the VRV and the VFC. Additionally, it was found that the power distribution between LF and HF in the VRV spectra was reversed with respect to heart rate spectra. However, the physiological origins of these components are only partially explained and the interpretation of the spectral parameters resulting from RT sequences remains incomplete. From a methodological point of view, Multiple Correspondence Analysis (MCA) appears as an interesting tool to interpret several populations and explore relationships between heart rate and ventricular repolarization phenomena. These two last points are exactly the objective of this work

Materials and methods used in this study are briefly presented in section 2; protocols, parameters and the MCA method employed are described succinctly. Three MCA are then described and discussed in section 3: the two first MCA were applied to HRV and VRV respectively for different sportive disciplines; the third MCA studies the relationships between HRV and VRV spectral parameters.

\section{Methods}

\subsection{Study protocol}

From a previous study [3], a database of twenty-eight young healthy subjects (12 swimmers, 7 judokas and 9 sedentary subjects; 18 men, mean age of all subjects $20 \pm$ 2 years) was constructed. Athletes were instructed to perform 2 stress tests. Test A was held during the basic training (endurance period), and test $\mathrm{B}$ was held during the training period before competition (tapering period). The sedentary subjects undertook only test A. In total, 47 ECG records were analyzed. Each record is composed of 3 five minutes stages performed under the following conditions:

- Stage 1: free ventilation in supine position. 
- Stage 2: controlled ventilation in supine condition.

- Stage 3: free ventilation in standing position

RR sequences were obtained using the Grizali QRS detector followed by a manual verification of each detected R-wave [4]. RT series were obtained by applying the algorithm proposed by Vila et al [5]. Artifacts in the RT sequence were also manually removed. The RR and RT sequences were linearly interpolated and uniformly sampled at $2 \mathrm{~Hz}$. A time-varying autoregressive modeling of the interpolated RR an RT sequences was performed by using a 25 seconds sliding window. Power spectral densities were estimated. The LF and HF bands were defined respectively by $[0.04-0.15 \mathrm{~Hz}]$ and by [0.15-0.4 $\mathrm{Hz}$ ] as proposed in [6]. For each stage of the protocol the following parameters were determined: RR (mean RR interval), RT (mean RT apex interval), $\mathrm{LF}_{\mathrm{RR}}, \mathrm{HF}_{\mathrm{RR}}$, $\mathrm{LF} /(\mathrm{LF}+\mathrm{HF})_{\mathrm{RR}}$ for $\mathrm{HRV}$ sequences, and $\mathrm{LF}_{\mathrm{RT}}, \mathrm{HF} \mathrm{F}_{\mathrm{RT}}$, $\mathrm{LF} /(\mathrm{LF}+\mathrm{HF})_{\mathrm{RT}}$ for VRV sequences.

\subsection{Multiple correspondences analysis}

MCA is a descriptive technique designed to analyze simple multi-way tables containing a measure of correspondence between rows and columns [7]. MCA allows to study a population of $\boldsymbol{I}$ individuals described by $\boldsymbol{J}$ qualitative variables and to identify linear and non linear relationships between variables. MCA is performed on a Complete Disjunctive Table (CDT) where lines represent individuals and columns represent the variable's modalities or classes. At the intersection of line $i$ and column $\boldsymbol{k}$, we find $\boldsymbol{x}_{\boldsymbol{i} \boldsymbol{k}}$ which is worth 1 according to whether individual $\boldsymbol{i}$ has the modality or class $\boldsymbol{k}, 0$ if not.

In the HRV and VRV studies, variables are continuous. In order to create a CDT adapted to apply $\mathrm{MCA}$, the numerical variables to be analyzed $\left(\mathrm{LF}_{\mathrm{RR}}\right.$, $\left.\mathrm{HF}_{\mathrm{RR}}, \mathrm{LF} /(\mathrm{LF}+\mathrm{HF})_{\mathrm{RR}}, \mathrm{LF}_{\mathrm{RT}}, \mathrm{HF}_{\mathrm{RT}}, \mathrm{LF} /(\mathrm{LF}+\mathrm{HF})_{\mathrm{RT}}\right)$ have to be transformed in categorical qualitative variables. This transformation can be carried out by cutting the data into $C$ different (crisp) classes, according to their amplitude [7], but this approach presents two main drawbacks: 1) there is a loss of information when the numeric value of variable $\boldsymbol{q}$ is replaced by a value coding its membership to a class $\boldsymbol{j}$, thus, the original data cannot be reconstructed; 2) a discontinuity is created by this kind of coding; indeed if $\boldsymbol{b}_{\boldsymbol{k}}$ is the border which separates two intervals, a distance is artificially created between the points closer to both sides to $\boldsymbol{b}_{\boldsymbol{k}}$. One way to avoid this discontinuity is to make a progressive passage from one modality to another, by sharing a value closer to the border by the two modalities. This coding, known as fuzzy, is a particular kind of coding proposed by Gallego [8]. We have adopted this coding by choosing 7 modalities. After performing the MCA, individuals and variables are projected into the same factorial planes. To facilitate the interpretation, we sketch on the factorial planes a polygonal line that joints the different modalities, giving an idea of the global variable's behavior.

\section{Results}

\subsection{Study of the populations}

The first MCA (MCA1) was carried out taking all individuals described by the HRV parameters. The numerical variables $\left(\mathrm{RR}, \mathrm{LF}_{\mathrm{RR}}, \mathrm{HF}_{\mathrm{RR}}, \mathrm{LF} /(\mathrm{LF}+\mathrm{HF})_{\mathrm{RR}}\right)$ were transformed in categorical variables, as mentioned above.

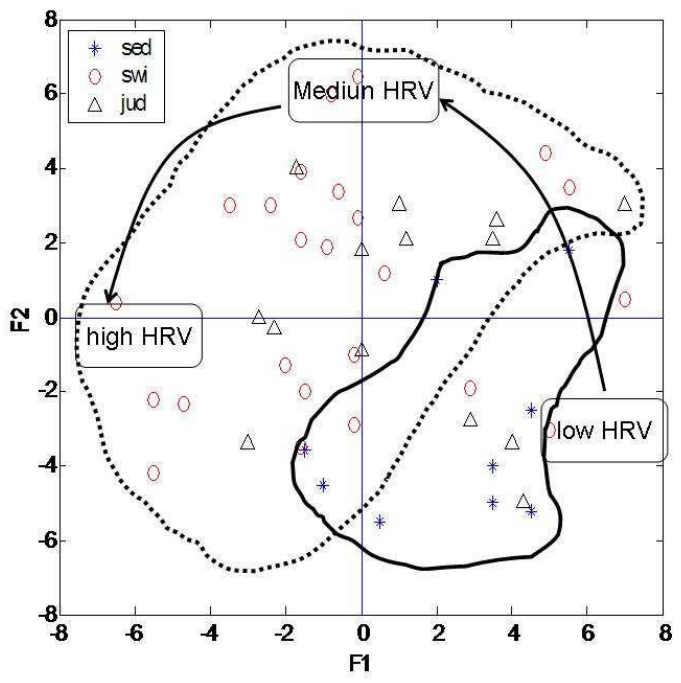

Figure 1. MCA1, study of the swimmers, judokas and sedentary populations through the HRV variables.

Results are schematized on figure 1, where subjects are shown on the first factorial plan (F1 vs F2). We observed that all variables were correlated, showing a Guttam effect. Classes showing the highest global heart rate variability values are located on the negative part of F1 and classes showing the lowest values are located on the positive part of F1. Sedentary subjects (sed) are close to the low-HRV class, and, in general, swimmers (swi) are related to the highest HRV parameters. Judokas (jud) occupy the whole first factorial plane.

ANOVA tests were performed on each axis. No significant difference was observed between sport seasons (endurance and tapering periods). We observed that swimmers and sedentary people are significantly different on both axes ( $\mathrm{p}<0.05$ in axis $1, \mathrm{p}<0.001$ in axis 2). Between sedentary and judokas, significant differences were only observed on the second axis $(\mathrm{p}<0.05)$. This difference is enhanced during the tapering period $(\mathrm{p}<0.01)$.

The second MCA (MCA2) was performed with the parameters of the VRV (RT, $\mathrm{LF}_{\mathrm{RT}}, \mathrm{HF}_{\mathrm{RT}}$, 
$\left.\mathrm{LF} /(\mathrm{LF}+\mathrm{HF})_{\mathrm{RT}}\right)$ ) also encoded in categorical variables. Results are schematized in figure 3. Variables and subjects are shown in superposition. Swimmers are related to the highest VRV values. The RT interval is shorter in sedentary subjects than in judokas and swimmers $(\mathrm{p}<0.001)$. This is a remarkable observation, because in exploring VRV parameters it is possible to separate all the judokas from sedentary, keeping the differences found in MCA1.

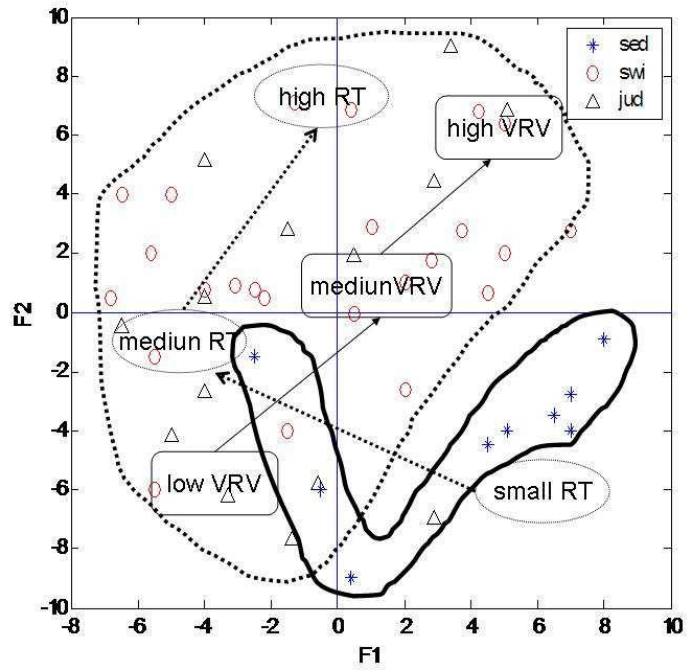

Figure 2. MCA2, study of the swimmers, judokas and sedentary populations through the VRV variables.

\subsection{Relationships between HRV and VRV spectral parameters}

By means of Boxplots, we have compared the ratios $\mathrm{LF} /(\mathrm{LF}+\mathrm{HF})_{\mathrm{RR}}$ and $\mathrm{LF} /(\mathrm{LF}+\mathrm{HF})_{\mathrm{RT}}$. We found that values for $\mathrm{LF} /(\mathrm{LF}+\mathrm{HF})_{\mathrm{RR}}$ are higher $(\mathrm{p}<0.001)$ than $\mathrm{LF} /(\mathrm{LF}+\mathrm{HF})_{\mathrm{RT}}$ in all stages. Generally, $\mathrm{LF} /(\mathrm{LF}+\mathrm{HF})_{\mathrm{RR}}$ is higher than 0.5 and $\mathrm{LF} /(\mathrm{LF}+\mathrm{HF})_{\mathrm{RT}}$ is smaller than this value (figure 3). Reversal Power distribution between HRV and VRV was verified [2].

Lastly, in a third MCA (MCA3), we explored the relationships between spectral parameters of $\mathrm{HRV}$ and VRV. Results are schematized in figure 4, representing the trajectories of each modality. Continuous lines represent parameters from HRV and dotted lines represent parameters from VRV. Line origins represent the weakest modality of each parameter and the arrow indicates the strongest modality.
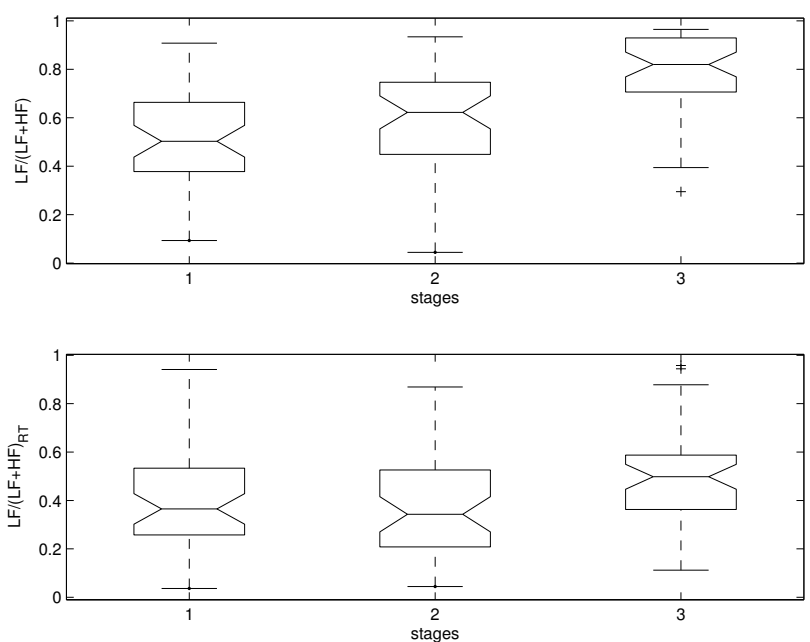

Figure 3. Reversal power for HRV and VRV.

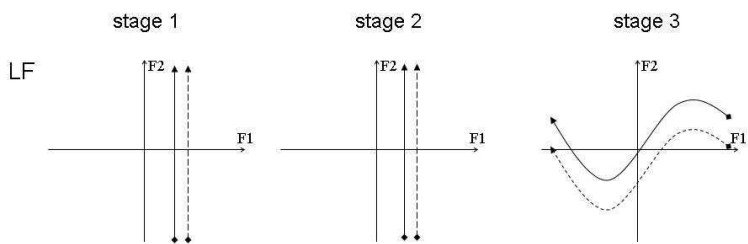

HF
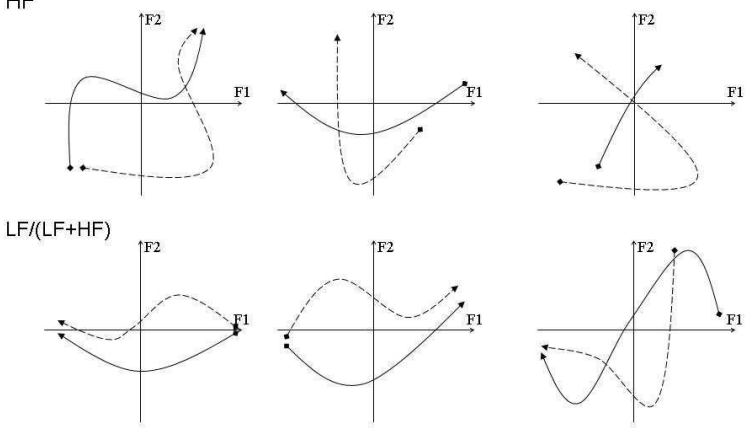

Figure 4. MCA3, behavior of HRV and VRV spectral variables in the first factorial plan for free ventilation in supine condition (stage 1), controlled ventilation (stage 2) and free ventilation in standing condition (stage 3 ).

From this schema we can draw the following observations for each parameter:

- LF: values from HRV and VRV series are correlated at all stages.

- HF: At the first stage, extremes modalities (smallest and largest) of $\mathrm{HF}_{\mathrm{RR}}$ and $\mathrm{HF}_{\mathrm{RT}}$ present similar values, but mean modalities from HRV are negative in axis 1 and positive in axis 2 while VRV values are in opposition. During the second stage (controlled ventilation in supine condition) $\mathrm{HF}$ is on axis 1 , while $\mathrm{HF}_{\mathrm{RT}}$ is on axis 2 . At third stage $\mathrm{HF}_{\mathrm{RR}}$ shows less variability than $\mathrm{HF}_{\mathrm{RT}}$. 
- LF/(LF+HF): during the first and second stages, extreme values are correlated but mean values are in opposition. At the third stage, only the large values are correlated.

\section{Discussion and conclusions}

These analyses give us very interesting results. From a classification viewpoint, we could make differences between the studied populations: $i$ ) the aerobic discipline (swimming) presented more variability than the anaerobic discipline (judo) and than sedentary people $i$ ) mean RT interval are longer for sportsmen whatever the discipline (aerobic or aneorobic), iii) no significant difference were observed between the training and the tapering periods in swimmers and judokas sportsmen. It is notable to observe that when we analyzed VRV parameters (MCA2) we obtained more information than with HRV parameters. This can be explained by the fact that VRV is modulated by HRV and because the RT interval duration is a powerful index.

MCA made possible to observe the relationship between LF from HRV and VRV series in the three physiological conditions. On the other hand, no clear relationship was observed for the high frequencies, only extreme values were correlated. Consequently, the $\mathrm{LF} /(\mathrm{LF}+\mathrm{HF})$ ratio obtained from VRV cannot be interpreted in the same manner as in HRV since we did not find any linear or nonlinear relationship between high frequencies parameters of VRV and HRV series. However, high frequencies show a certain correlation in supine position that cannot be observed in the other stages. Parameters related to high frequencies of the RT space have to be considered with care when characterizing the ANS modulation on ventricular repolarization.

Our findings are in agreement with previous studies $[9,10]$ that have shown that most of the ventricular repolarization variability is due to the modulation of heart rate variability and that slow components of RT and RR sequences correlate better than fast ones.

In this study, preliminary exploratory results were shown; MCA have shown to be a powerful tool to elucidate how heart rate modulation and ANS operate in ventricular repolarization. Moreover, this method would permit to study how repolarization dynamicity could be used as an index of arrhythmia risk. These questions remain challenging issues.

\section{Acknowledgements}

This work has been supported in part by the DID University Simón Bolívar and by ECOS NORD cooperation program.

Thanks to Dr. Jean-Yves BANSARD for his help in MCA comprehension.

\section{References}

[1] Merri M., Alberti M., Moss A. Dynamic analysis of ventricular repolarization duration from 24-hour holter recordings. IEEE Trans Biomed Eng 1993, 40(12):1219-25

[2] Nollo G. Speranza G., Grasso R., Bonamini R., Mangiardi L., Antolini R. Spontaneous beat-to beat variability of the ventricular repolarization duration. J. Electrocardiology 1992, 25(1) 9-17.

[3] Wong S., Gauvrit H., Cheaib N., Carré F., Carrault G. Multiple Factor Analysis as a tool for studying the effect of physical training on the autonomic Nervous System. In Computers in Cardiology 2002. Computer Society Press. 2002

[4] Gritzaldi F. Towards a generalized scheme for QRS detection in ECG waveforms. Signal Processing, 1988, 15:183-92

[5] Vila J. Gang Y., Rodríguez J., Fernández M., Barro D., Malik M. A new approach for TU complex characterization. IEEE Trans Biomed Eng, 2000 47(6):76472

[6] Task Force of the European Society of Cardiology and the North American Society of Pacing and Electrophysiology. Heart rate variability: standard of measurement, physiological interpretation and clinical use. Circulation 1996;93(5):1043-65

[7] Escofier B., Pagés J. Analyses Factorielles simples et multiples: objectifs, méthodes et interprétation. 1988, Paris, Dunod.

[8] Gallego F. Codage flou en analyse des correspondances. Les cahiers de l'analyse de données, 1982, VII (4) :413430

[9] Porta A., Baselli G., Caiani E., Malliani A., Lombardi F., Cerutti S. Quantifying electrocardiogram RT-RR variability interactions. Med Biol Eng Comput, 1998, 36(1):27-34

[10] Marciano F., Coumo S., Migaux M., Vetrano A., Dynamic Correlation between QT and RR Intervals: How Long is QT adaptation to heart rate? Computers in Cardiology 1998 413-16

Address for correspondence

Sara Wong

Departamento de Electrónica y Circuitos

Universidad Simón Bolívar

Caracas Venezuela

swong@usb.ve 